\title{
Amenorrhea Induced By Chemotherapy In Premenopausal Breast Cancer Patients (Retrospective Study)
}

\author{
El-Sayed Sadoon ${ }^{1, *}$ MSc, Hassan Hamdy ${ }^{1}$ MD, Hesham Al-Abady ${ }^{1}$ MD
}

*Corresponding Author:

El-Sayed Sadoon

Drsayed6445@gmail.com

Received for publication January 7, 2020; accepted January 24, 2020; published on line January 26, 2020.

Copyright 2020 The Authors published by Al-Azhar University, Faculty of Medicine, Cairo, Egypt. All rights reserved. This an openaccess article distributed under the legal terms, where it is permissible to download and share the work provided it is properly cited. The work cannot be changed in any way or used commercially.

doi:10.21608/aimj.2020.22107. 1058

${ }^{1}$ Clinical Oncology \& Nuclear Medicine Department, Faculty of Medicine, Al-Azhar University, Cairo Egypt.

\begin{abstract}
Background: Breast cancer is the most frequently diagnosed cancer globally and is the leading cause of cancer-related death in women. The American Cancer Society estimates that 249, 260 Americans will be diagnosed with invasive breast cancer. Objectives: This is a retrospective study aiming to identify the frequency of amenorrhea (ovarian failure) after different chemotherapy regimens in premenopausal patients with breast cancer as well as the associated other risk factors that could influence the rate of amenorrhea. Patients and Methods: This study will include patients with breast cancer with at least a minimum follow up of six months after last cycle of chemotherapy either clinically or by E2 and FSH. Expected number of enrolled patients in this study is around 100 patients. Results: Patients who had HER 2 negative IHC stain had significantly lower rates of Chemotherapy Induced Amenorrhea (CIA) when compared to those HER 2 positives with $p$ value 0.001 . In our study we have found a correlation between DFS and CIA which imply that patients who had CIA had better survival than those with CIA however this difference was not significant, $p$ value 0.129 , Overall survival show no difference based on CIA $p$ value 0.319 . Conclusion: CIA is a main concern for females treated with breast cancer, it was found correlated to age, tumor stage, tumor biology and type of surgery. More prospective studies are needed to confirm correlation between CIA and prognosis of breast cancer, nonetheless other treatment regimens and malignancies should be investigated.
\end{abstract}

Keywords: Chemotherapy; Amenorrhea; Premenopausal Breast Cancer Patients.

\section{INTRODUCTION}

Breast cancer is the most frequently diagnosed cancer globally and is the leading cause of cancerrelated death in women. The American Cancer Society estimates that 249,260 Americans will be diagnosed with invasive breast cancer and 40, 890 will die of the disease in the United States in $2016 .{ }^{1}$

The treatment of breast cancer includes the treatment of local disease with surgery, radiation therapy, or both, and systemic treatment with chemotherapy, endocrine therapy, biologic therapy, or combinations of these. The need for and selection of various local or systemic therapies are based on several prognostic and predictive factors. These factors include tumor histology, clinical and pathologic characteristics of the primary tumor, ALN status, tumor hormone receptor (ER \& PR) content, tumor HER2 status. Adjuvant endocrine therapy is an integral component of care for endocrine-dependent breast cancer (EDBC). The goal of this type of therapy is to counteract the production and the action of oestrogens. $^{2}$

The ovaries of premenopausal women who received chemotherapy typically have normal to mildly decreased numbers of primordial follicles and a greater decrease in the numbers of maturing follicles,
Authorship: All authors have a substantial contributions to the article.

Disclosure: The authors have no financial interest to declare in relation to the content of this article. The Article Processing Charge was paid for by the authors.

indicating a greater effect on follicular development than on oocytes. Consistent with these histologic findings are the clinical observations that many women are amenorrhoeic during chemotherapy, often with high serum gonadotrophin concentrations, but that menstrual function and fertility often return several months to years after the cessation of therapy. $^{3}$

Chemotherapy-induced amenorrhea is a wellrecognized side effect of cytotoxic chemotherapy. These drugs appear to have a greater effect on follicular development than on oocytes, with variable degrees of subsequent ovarian dysfunction. Some women develop complete follicular depletion and permanent ovarian failure during chemotherapy, while others may become amenorrhoeic during therapy, but then have subsequent return of ovarian function, menstrual cycles, and fertility, months to years after the cessation of therapy. There are wellrecognized risk factors for chemotherapy-induced 
amenorrhea or ovarian failure, such as age, chemotherapy regimen, subsequent use of endocrine therapy and genetics. ${ }^{4}$

\section{PATIENT AND METHODS}

This retrospective study included all patients with breast cancer with at least a minimum follow up of six months after last cycle of chemotherapy either clinically or by $\mathrm{E}_{2}$ and FSH. Number of patients enrolled in this study is 97 patients from January 2009 to December 2015.

Inclusion criteria: Female patient, Age $>18$ years, pathologically confirmed breast cancer, Premenopausal at the diagnosis of breast cancer, Breast cancer at any stage and Six month or more follow up after last cycle of chemotherapy.

Exclusion criteria: Postmenopausal women, Patient with double malignancy, Hysterectomy and Any kind of oophorectomy.

The following data collected from patients' files: Personal data (age, sex, marital state, sibling), Menstrual history (menarche, regularity), comorbidities (diabetes, hypertension, thyroid dysfunction), contraceptive history, disease criteria (date of first diagnosis، extent of disease, histological type, stage), treatment related data (chemotherapy regimen, number of cycles' regularity), amenorrhea related data (time of amenorrhea, reversibility).

\section{ETHICAL APPROVAL:}

Informed consents were obtained from all participants during their follow up at clinic, and data confidentiality was maintained throughout the study and any resulting publication anonymously.

\section{STATISTICAL METHODS:}

Amenorrhea defined as absence of menstruation in a woman of reproductive age for more than 6 months either clinically or by $\mathrm{E}_{2} 20<, \mathrm{FSH}>20$. Data presented as mean and standard deviation or median and range as appropriate. Categorical data will be presented as frequency and percentage. Relation between categorical data done using or appropriate statistical test. For quantitative data comparison between two groups done using either parametric or non parametric $\mathrm{t}$ - test. $\mathrm{P}$-value less than 0.05 will be considered significant.

\section{RESULTS}

\section{Patient's characteristics:}

The mean age of all patients was 41.3 years ranges between (28-54) years. Out of these patients, 93 $(95.9 \%)$ were married while 4 patients $(4.1 \%)$ were single. Seventy-seven patients $(79.4 \%)$ gave history of regular menses while $16(21.6 \%)$ patients had history for irregular menstruation. Table (1), Figure (1)

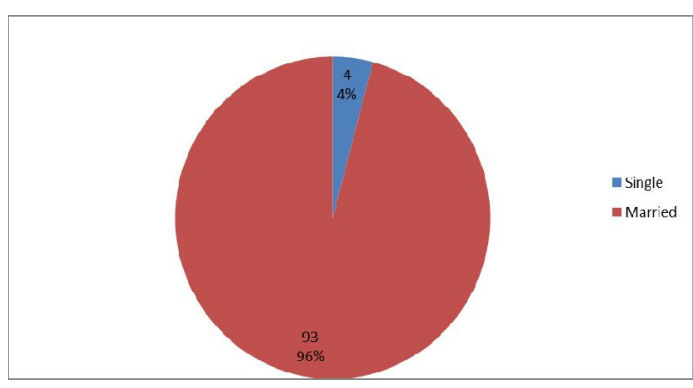

Fig. 1: Pie diagram shows marital status of patients included in the current study.

Diabetes was observed in 6 patients $(6.2 \%)$ and 10 patients were hypertensive $(10.3 \%)$, while 3 patients $(3.1 \%)$ were diagnosed with thyroid disorders. Table (1), Figure (2)

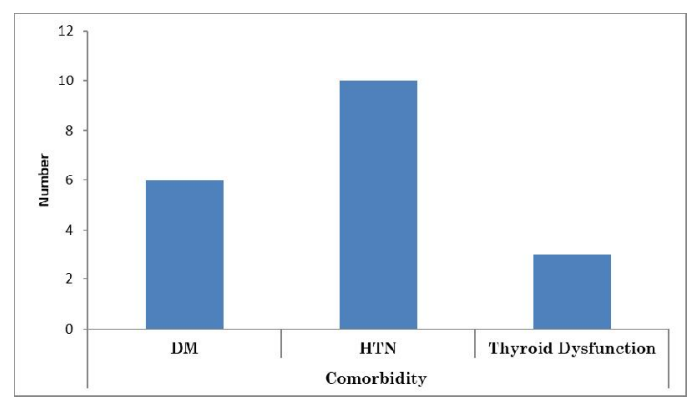

Fig. 2: Histogram shows comorbidities of patients included in the current study.

\section{Histopathological characteristics:}

Invasive duct carcinoma diagnosed in 93 patients (95.8\%), invasive lobular carcinoma also diagnosed in 2 patients $(2.1 \%)$, while 2 patients $(2.1 \%)$ diagnosed with mucinous variant. Table (2), Figure (3)

MRM done for 55 patients (56.7\%) while conservative breast surgery done for the rest of the patients (43.3\%). Figure (4)

Eight patients $(8.2 \%)$ presented with $\mathrm{T} 1$ staging, T2 staging found in 60 patients $(61.9 \%)$, and 19 patients $(19.6 \%)$ had T3 staging, while 10 patients $(10.3 \%)$ had T4B disease. Table (2)

Positive estrogen receptors found in 82 patients $(84.5 \%)$. while rest of patients $(15.5 \%)$ were negative. Positive progesterone receptor found in 77 patients $(79.4 \%)$, while rest of patients $(20.6 \%)$ were negative. Her2neu status was over expressed in 21 patients $(21.6 \%)$. While 77 patients $(78.4 \%)$ were negative. Table (2), Figure (5) 


\begin{tabular}{|c|c|c|c|}
\hline & & Number & $\%$ \\
\hline \multirow{2}{*}{ Age (years) } & Mean \pm SD & \multicolumn{2}{|c|}{$41.3 \pm 5.7$} \\
\hline & Range & \multicolumn{2}{|c|}{$28-54$} \\
\hline \multirow{2}{*}{ Marital Status } & Single & 4 & 4.1 \\
\hline & Married & 93 & 95.9 \\
\hline \multirow{2}{*}{ Menarche age } & Mean \pm SD & \multicolumn{2}{|c|}{$\begin{array}{l}1 \\
12.7 \pm 1.0\end{array}$} \\
\hline & Range & \multicolumn{2}{|c|}{$11-14$} \\
\hline \multirow[b]{2}{*}{ Menstrual Regularity } & Yes & 77 & 79.4 \\
\hline & No & 16 & 21.6 \\
\hline Siblings & Median (Range) & \multicolumn{2}{|c|}{$3(0-6)$} \\
\hline \multirow{2}{*}{ Contraception* } & Hormonal & 41 & 42.3 \\
\hline & IUD & 52 & 53.6 \\
\hline \multirow{3}{*}{ Comorbidity } & DM & 6 & 6.2 \\
\hline & HTN & 10 & 10.3 \\
\hline & Thyroid Dysfunction & 3 & 3.1 \\
\hline \multirow{2}{*}{ Family History } & No & 82 & 84.5 \\
\hline & Yes & 15 & 15.5 \\
\hline
\end{tabular}

*among married

Table 1: Patient characteristics

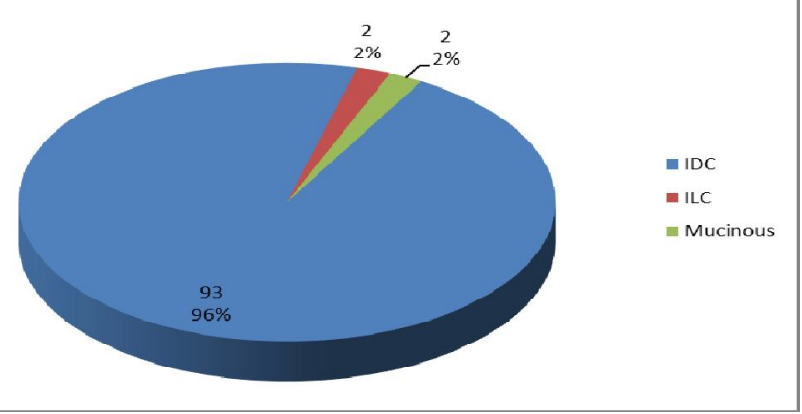

Fig. 3: Pie diagram shows histopathological characteristics of tumors included in the current study.

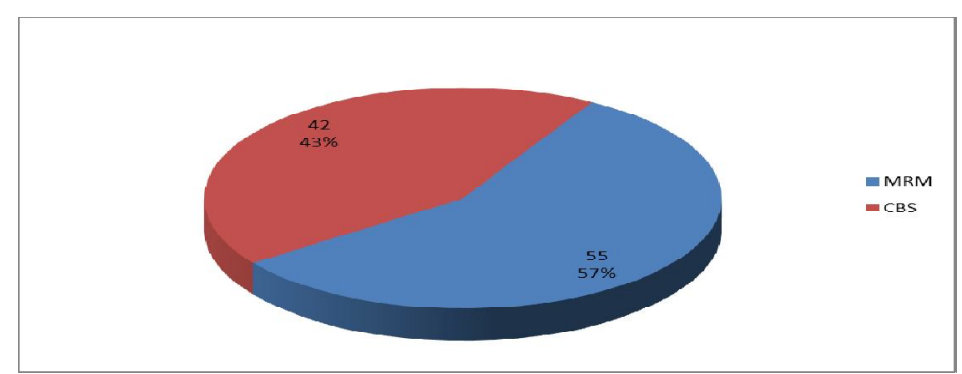

Fig. 4: Pie diagram shows type of surgery done for patients included in the current study. 


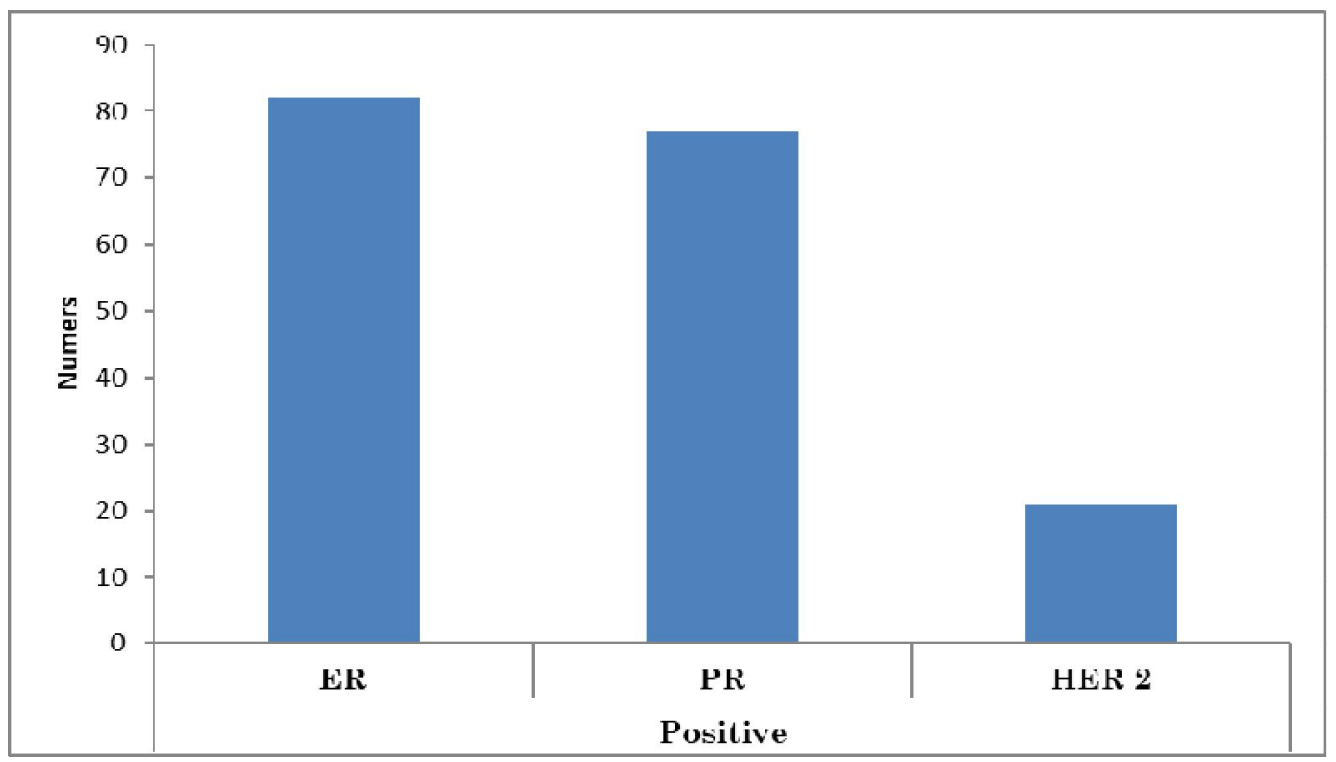

Fig. 5: Histogram shows immunophenotyping pattern of study populations.

\begin{tabular}{|c|c|c|c|}
\hline & & Number & $\%$ \\
\hline \multirow{2}{*}{ Surgery } & MRM & 55 & 56.7 \\
\hline & CBS & 42 & 43.3 \\
\hline \multirow[t]{4}{*}{ T Stage } & T1 & 8 & 8.2 \\
\hline & $\mathrm{T} 2$ & 60 & 61.9 \\
\hline & $\mathrm{T} 3$ & 19 & 19.6 \\
\hline & T4B & 10 & 10.3 \\
\hline \multirow[t]{4}{*}{ N Stage } & N0 & 30 & 30.9 \\
\hline & N1 & 33 & 34.0 \\
\hline & $\mathrm{N} 2$ & 27 & 27.8 \\
\hline & N3 & 7 & 7.2 \\
\hline \multirow[t]{3}{*}{ Histology } & IDC & 93 & 95.8 \\
\hline & ILC & 2 & 2.1 \\
\hline & Mucinous & 2 & 2.1 \\
\hline \multirow[t]{2}{*}{ Grade } & II & 95 & 97.9 \\
\hline & III & 2 & 2.1 \\
\hline \multirow[t]{2}{*}{ ER } & Negative & 15 & 15.5 \\
\hline & Positive & 82 & 84.5 \\
\hline \multirow[t]{2}{*}{$\mathbf{P R}$} & Negative & 20 & 20.6 \\
\hline & Positive & 77 & 79.4 \\
\hline \multirow[t]{2}{*}{ HER 2} & Negative & 76 & 78.4 \\
\hline & Positive & 21 & 21.6 \\
\hline
\end{tabular}

Table 2: Histopathological characteristics

\begin{tabular}{|c|c|c|c|c|c|c|c|c|c|c|c|}
\hline & \multicolumn{10}{|c|}{ Induced Amenorrhea } & \multirow[b]{3}{*}{ P value } \\
\hline & \multicolumn{5}{|c|}{ No } & \multicolumn{5}{|c|}{ Yes } & \\
\hline & Median & Min. & Max. & Q1 & Q3 & Median & Min. & Max. & Q1 & Q3 & \\
\hline LN Resected & 17 & 8 & 30 & 14 & 20 & 18 & 12 & 22 & 15 & 21 & 0.669 \\
\hline LN Positive & 2 & 0 & 20 & 0 & 5 & 3 & 0 & 10 & 2 & 6 & 0.030 \\
\hline Cycles \# & 6 & 4 & 8 & 4 & 8 & 6 & 3 & 8 & 6 & 8 & 0.411 \\
\hline E2 Level & 111.5 & 15 & 1680 & 45 & 230 & 14 & 5 & 19 & 10 & 19 & $<0.001$ \\
\hline FSH Level & 16 & 5 & 61 & 11 & 20 & 53 & 25 & 123 & 36 & 85 & $<0.001$ \\
\hline
\end{tabular}

Table 3: Induced amenorrhea 



\begin{tabular}{|c|l|c|c|c|c|}
\hline \multicolumn{2}{|c|}{ Induced Amenorrhea } & N & Mean & SD & P value \\
\hline \multirow{2}{*}{ Age } & No & 56 & 38.9 & 4.7 & $<0.001$ \\
\cline { 2 - 6 } & Yes & 32 & 45.0 & 4.3 & \\
\hline \multirow{2}{*}{ Menarche } & No & 56 & 12.5 & 0.9 & 0.064 \\
\cline { 2 - 6 } & Yes & 32 & 12.8 & 0.7 & \\
\hline \multirow{2}{*}{ Largest Size } & No & 55 & 4.3 & 1.5 & 0.259 \\
\cline { 2 - 6 } & Yes & 26 & 4.8 & 2.0 & \\
\hline
\end{tabular}

SD: standard deviation, $\mathrm{p} \leq 0.05$ is considered statistically significant, analysis done by independent $\mathrm{t}$ test

Table 4: Age of menarche

\begin{tabular}{|c|c|c|c|c|c|c|}
\hline & & \multicolumn{4}{|c|}{ Induced Amenorrhea } & \multirow[b]{3}{*}{ p value } \\
\hline & & \multicolumn{2}{|c|}{ No } & \multicolumn{2}{|c|}{ Yes } & \\
\hline & & Count & $\%$ & Count & $\%$ & \\
\hline \multirow{2}{*}{ Marital Status } & Single & 4 & 100.0 & 0 & 0.0 & 0.122 \\
\hline & Married & 52 & 61.9 & 32 & 38.1 & \\
\hline \multirow{2}{*}{ Regularity } & No & 6 & 30.0 & 14 & 70.0 & $<0.001$ \\
\hline & Yes & 50 & 73.5 & 18 & 26.5 & \\
\hline \multirow{3}{*}{ Contraception } & Hormonal & 29 & 70.7 & 12 & 29.3 & 0.035 \\
\hline & IUD & 19 & 48.7 & 20 & 51.3 & \\
\hline & Others-None & 4 & 100.0 & 0 & 0.0 & \\
\hline \multirow{2}{*}{ DM } & No & 55 & 67.1 & 27 & 32.9 & 0.013 \\
\hline & Yes & 1 & 16.7 & 5 & 83.3 & \\
\hline \multirow{2}{*}{ HTN } & No & 52 & 66.7 & 26 & 33.3 & 0.099 \\
\hline & Yes & 4 & 40.0 & 6 & 60.0 & \\
\hline \multirow{2}{*}{ Thyroid Dysfunction } & No & 53 & 62.4 & 32 & 37.6 & 0.183 \\
\hline & Yes & 3 & 100.0 & 0 & 0.0 & \\
\hline \multirow{2}{*}{ Family History } & No & 50 & 65.8 & 26 & 34.2 & 0.291 \\
\hline & Yes & 6 & 50.0 & 6 & 50.0 & \\
\hline \multirow{2}{*}{ Surgery } & MRM & 23 & 46.9 & 26 & 53.1 & $<0.001$ \\
\hline & CBS & 33 & 84.6 & 6 & 15.4 & \\
\hline \multirow{3}{*}{ Histology } & IDC & 52 & 61.9 & 32 & 38.1 & 0.302 \\
\hline & ILC & 2 & 100.0 & 0 & 0.0 & \\
\hline & Mucinous & 2 & 100.0 & 0 & 0.0 & \\
\hline \multirow{2}{*}{ Grade } & II & 54 & 62.8 & 32 & 37.2 & 0.532 \\
\hline & III & 2 & 100.0 & 0 & 0.0 & \\
\hline \multirow{2}{*}{ ER } & Negative & 3 & 50.0 & 3 & 50.0 & 0.472 \\
\hline & Positive & 53 & 64.6 & 29 & 35.4 & \\
\hline \multirow{2}{*}{ PR } & Negative & 8 & 72.7 & 3 & 27.3 & 0.503 \\
\hline & Positive & 48 & 62.3 & 29 & 37.7 & \\
\hline \multirow{2}{*}{ HER 2} & Negative & 51 & 72.9 & 19 & 27.1 & $<0.001$ \\
\hline & Positive & 5 & 27.8 & 13 & 72.2 & \\
\hline \multirow{4}{*}{ T Stage } & $\mathrm{T} 1$ & 6 & 75.0 & 2 & 25.0 & 0.003 \\
\hline & $\mathrm{T} 2$ & 38 & 70.4 & 16 & 29.6 & \\
\hline & T3 & 11 & 68.8 & 5 & 31.3 & \\
\hline & T4B & 1 & 10.0 & 9 & 90.0 & \\
\hline \multirow{4}{*}{ N Stage } & N0 & 19 & 79.2 & 5 & 20.8 & 0.217 \\
\hline & N1 & 21 & 63.6 & 12 & 36.4 & \\
\hline & N2 & 14 & 51.9 & 13 & 48.1 & \\
\hline & N3 & 2 & 50.0 & 2 & 50.0 & \\
\hline
\end{tabular}

$\mathrm{p} \leq 0.05$ is considered statistically significant, analysis done by Chi square test $\mathrm{t}$ stage t4B is statistically significant from other stages.

Table 5: Factors in relation with amenorrhea status. 


\begin{tabular}{|c|c|c|c|c|c|c|}
\hline \multirow{2}{*}{ Factors } & \multicolumn{4}{|c|}{ DFS \% } & \multirow{2}{*}{$\frac{\text { Median(months) }}{(95 \% \mathrm{CI})}$} & \multirow[b]{2}{*}{ p value } \\
\hline & $\mathbf{n}$ & 2 years & 3 years & 5 years & & \\
\hline All & 97 & 96.9 & 90.6 & 85.0 & 82.3 & $\mathrm{NA}$ \\
\hline \multicolumn{7}{|l|}{ Age } \\
\hline$\leq 40$ & 48 & 95.8 & 93.2 & 84.7 & 82.3 & \multirow[t]{2}{*}{0.879} \\
\hline$>40$ & 49 & 97.9 & 88.1 & 80.7 & NA & \\
\hline \multicolumn{7}{|l|}{ Marital Status } \\
\hline Single & 4 & 100 & 100 & 100 & NA & 0.467 \\
\hline Married & 93 & 96.8 & 90.3 & 84.4 & NA & \\
\hline \multicolumn{7}{|l|}{ Siblings } \\
\hline$\leq 3$ & 60 & 96.6 & 90.7 & 87.5 & 82.4 & 0.933 \\
\hline$>3$ & 33 & 97.0 & 89.4 & 78.8 & NA & \\
\hline \multicolumn{7}{|l|}{ Menarche Age } \\
\hline$<13$ & 34 & 100 & 100 & 94.4 & NA & 0.023 \\
\hline$\geq 13$ & 63 & 95.2 & 86.0 & 80.4 & $82.3(65.4-99.3)$ & \\
\hline \multicolumn{7}{|l|}{ Regular Cycles } \\
\hline No & 20 & 100 & 93.8 & 82.0 & $\overline{\mathrm{NA}}$ & 0.604 \\
\hline Yes & 77 & 96.0 & 89.9 & 85.3 & $82.4(82.0-82.9)$ & \\
\hline \multicolumn{7}{|l|}{ Contraception } \\
\hline Hormonal & 41 & 100 & 100 & 94.7 & NA & 0.009 \\
\hline IUD & 48 & 93.7 & 85.0 & 73.2 & 82.3 & \\
\hline Others-none & 4 & 100 & 100 & 100 & NA & \\
\hline \multicolumn{7}{|l|}{$\mathbf{D M}$} \\
\hline No & 91 & 96.7 & 91.6 & 85.6 & 82.3 & 0.520 \\
\hline Yes & 6 & 100 & 75.0 & NA & NA & \\
\hline \multicolumn{7}{|l|}{ HTN } \\
\hline No & 87 & 96.5 & 89.7 & 83.7 & NA & 0.194 \\
\hline Yes & 10 & 100 & 100 & 100 & NA & \\
\hline \multicolumn{7}{|c|}{ +ve Family history } \\
\hline No & 82 & 97.6 & 90.2 & 85.8 & 82.4 & 0.885 \\
\hline Yes & 15 & 92.9 & 92.9 & 79.6 & NA & \\
\hline \multicolumn{7}{|l|}{ Surgery } \\
\hline MRM & 55 & 94.5 & 83.8 & 77.4 & $82.3(40.8-123.9)$ & 0.054 \\
\hline CBS & 42 & 100 & 100 & 95.5 & NA & \\
\hline \multicolumn{7}{|l|}{ Stage } \\
\hline Early & 68 & 98.5 & 98.3 & 92.7 & NA & 0.001 \\
\hline Late & 29 & 93.1 & 73.5 & 67.9 & 82.3 & \\
\hline \multicolumn{7}{|l|}{ Size } \\
\hline$<4.5$ & 41 & 97.1 & 97.1 & 92.6 & $82.3(52.9-111.7)$ & 0.684 \\
\hline$\geq 4.5$ & 49 & 98.0 & 91.2 & 84.0 & 88.6 & \\
\hline
\end{tabular}

$\mathrm{P} \leq 0.05$ is statistically significant, $\mathrm{CI}$ : confidence interval, NA: not applicable

Table 6: Disease free survival among all patients' groups

\section{DISCUSSION}

Breast cancer is the commonest malignancy affecting females worldwide, it is estimated to be the fifth cause of death in cancer related mortalities. Breast cancer is a systemic disease, meaning that it require systemic treatment as main line of therapy, chemotherapy is the key player in adjuvant treatment of breast cancer patients, may regimens has been discussed in literature proving that Anthracycline based chemotherapy lead to best prognosis, in addition to paclitaxel it has improved the OS and DFS in patients with advanced stages. ${ }^{5}$

Chemotherapy induced amenorrhea and fertility preservation has been a critical point of discussion since $^{6,7}$ found that $41 \%$ of breast cancer survivors experienced amenorrhea during treatment, while Melanie and colleagues confirmed that $31 \%$ of their sample had amenorrhea during chemotherapy treatment, in our study $33 \%$ had chemotherapy induced amenorrhea.

In the current study, age was found to be statistically correlated to chemotherapy induced amenorrhea as patients with older ages are more borne for permanent amenorrhea and ovarian failure while younger ages were more borne to transient amenorrhea with $\mathrm{p}$ value 0.0001 , any authors have confirmed same results. ${ }^{8,9}$ 
It is believed that regularity of menses is correlated with induction of amenorrhea post chemotherapy as detailed by ${ }^{10}$ patients who had a Hodgkin's disease with irregular menses experienced longer amenorrhea period than those with regular one, in our study patients with regular menses had lower incidence of chemotherapy induced amenorrhea with $\mathrm{p}$ value 0.001 when compared to those with irregular menses. ${ }^{10}$

Our study was the first to find a statistically significant correlation between chronic disease as DM and CIA with $p$ value 0.013 when compared to patients who didn't suffer from DM, nonetheless we discovered that patients who had CBS had lower rates of CIA, this may be due to younger women tend to have conservative surgeries to maintain the cosmetic contour of the breast while older women usually go for MRM to avoid adjuvant chemotherapy and postoperative radiation $\mathrm{p}$ value 0.001 .

Knowing that in our sample patients who had HER 2 negative IHC stain had significantly lower rates of CIA when compared to those HER 2 positive stain with $\mathrm{p}$ value 0.001 . In the current study, we have found a correlation between DFS and CIA which imply that patients who had CIA had better survival than those with CIA however this difference was not significant, $p$ value 0.129 , Overall survival show no difference based on CIA $p$ value 0.319 .

Many limitations have been faced in our study, being a retrospective study, it had missed data and incomplete follow up records, larger prospective studies are needed to confirm prognosis of CIA on patient's survival, as well as investigation of other protocols and malignancies.

\section{CONCLUSION}

CIA is a main concern for females treated with breast cancer, it was found correlated to age, tumor stage, tumor biology and type of surgery. More prospective studies are needed to confirm correlation between CIA and prognosis of breast cancer, nonetheless other treatment regimens and malignancies should be investigated.

\section{REFERENCES}

1. Siegel R, De Santis C, Virgo K, et al. Cancer treatment and survivorship statistics. CA Cancer $J$ Clin; 2012; 62(4): 220-41.

2. Jankowitz C, Mc Guire P and Davidson E. Optimal systemic therapy for premenopausal women with hormone receptor-positive breast cancer. The Breast.; 2013; 22: S165-70.

3. Steven C. Patterns of relapse and long-term complications of therapy in breast cancer survivors UpToDate Journal,2013. Available at:

https://www.uptodate.com/contents/patterns-ofrelapse-and-long-term-complications- of-therapy-inbreast-cancer-survivors.

4. Shapiro CL. Acute side effects of adjuvant chemotherapy for early stage breast cancer. In D. S. Basow (Ed.), UpToDate. Waltham, MA: UpToDate; 2013. Available at: https://humannaturenaturalhealth.com/cancercare?ke yword session $\mathrm{id}=\mathrm{vt} \sim$ adwords $\mid \mathrm{kt} \% 2 \mathrm{~B}$ for $\% 20 \% 2 \mathrm{~B}$ breast $\% 20 \% 2$ Bcancer|mt $\sim$ b|ta $264084789297 \&$ vsr efdom=wordstream\&gclid $=$ Cj0KCQiA9orxBRD0A RIsAK9JDxSEYLAbamdy2QL0kDll4VzklcuWUhF QMWe2puet6IzlyEiNZPePDTQaAtf3EALw wcB.

5. Ball J. and Fox K. Age-associated incidence of chemotherapy-related amenorrhea (CRA) following adjuvant doxorubicin, cyclophosphamide, and paclitaxel (AC/TAXOL) in early-stage breast cancer. Proc Am Soc Clin Oncol 2001; 20:16B, (abstr 1810).

6. Fisher B, Ravdin G, Ausman K et al. Surgical adjuvant chemotherapy in cancer of the breast: results of a decade of cooperative investigation. Annals of surgery, 1968; 168(3), 33756.

7. Sukumvanich P, Case D, Van Zee K, et al., Incidence and time course of bleeding after longterm amenorrhea after breast cancer treatment: a prospective study. Cancer; 2010; 116: 3102-11.

8. Melanie H, Jacobson D, Ann C, et al. Menses resumption after cancer treatment - induced amenorrhea occurs early or not at all. Fertility and Sterility; 2015; 105(3):765-72.

9. Letourneau M, Ebbel E, Katz P, et al. Acute ovarian failure underestimates age-specific reproductive impairment for young women undergoing chemotherapy for cancer. Cancer; 2012; 118:1933-9.

10. Horning J, Hoppe T, Kaplan S. Et al. Female reproductive potential after treatment for Hodgkin's disease. N Engl J Med; 1981;304: 1377-82. 